\title{
P476: Long-term pathology of the naturally-acquired Pneumocystis primary infection in rats
}

\author{
Ponce C.A. ${ }^{1}$, Bustamante R. ${ }^{1}$, Iturra P.A. ${ }^{1}$, Rojas D.A. ${ }^{1}$, Méndez A. ${ }^{1}$, Vargas SL. ${ }^{1}$
}

${ }^{1}$ Biomedical Sciences Institute, University of Chile School of Medicine, Santiago, Chile

\section{INTRODUCCION}

- Pneumocystis is the most common pulmonary infection in healthy infants, with a peak incidence between 2 and 5 months of age that is not diagnosed.

- Recently, we have shown in an animal model that the primary infection induces lung damage consisting of increased mucus, airway epithelial thickening, perivascular and peribronchial infiltrates, and increased collagen that suggests chronic changes secondary to Pneumocystis in immunocompetent rats up to day 75 post-infection.

- Whether the primary infection by Pneumocystis induces longlasting pathology is unknown and may suggest a role of this fungal infection in chronic airway disease. Therefore, we extended our evaluations in the same model until the age of 1 year.

\section{METHODS}

- 120 newborn rats kept in HEPA-filtered air containers throughout the experiment were randomized to exposure at birth to rats with Pneumocystis pneumonia by co-habitation for 48 hours, and to non-exposure. Non-exposed rats received anti-Pneumocystis prophylaxis (TMP/SMZ) for the duration of the experiment.

- 12 rats per group were sacrificed each time under deep anesthesia at 40,60, 80, 258 and 361 days of age. Half (6) received vascular perfusion with $3 \%$ buffered formalin via inferior cava vein, and their fixed lungs sectioned longitudinally separating central from distal airway lung zones for histology determinations according to Figs $2 \& 3$ using Image Pro-plus software. The other 6 rats were exsanguinated and their lungs removed, placed in RNA-later, and frozen fresh at $-80^{\circ} \mathrm{C}$ until used for Pneumocystis and collagen determinations.

- Pneumocystis was diagnosed using n-PCR with specific primers that amplifying the mtLSUrRNA, and collagen levels were biochemically determined by hydroxyproline method.
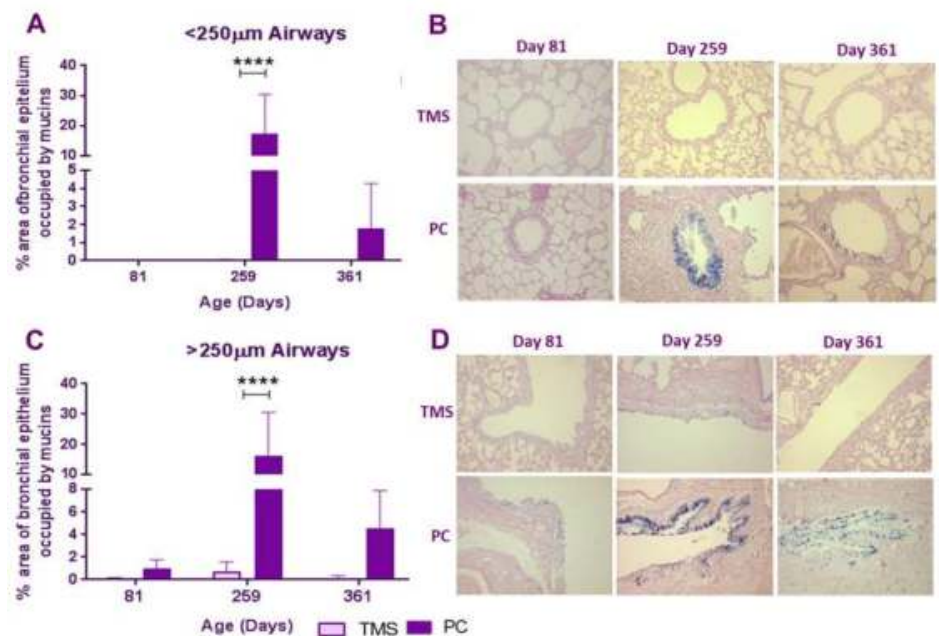

Figure 3: (A) (C) Mucus content (\% epitelium area positive for Alcian blue-PAS) was assesed in rats at $81,259 \& 361$ days of age. (B) (D) Representative images showing the progression of mucus within of the epithelium.

\section{Funding}

FONDECYT Chile Grant 1140412 \& ERANet-LAC (HID0254)

\section{RESULTS}

- Pneumocystis-DNA amplified in 6/6(100\%), 6/6(100\%), $6 / 6(100 \%), 2 / 6(33 \%), 1 / 6(17 \%)$ of exposed rats and in none $(0 \%)$ of non-exposed rats at $40,60,81,258$ \& 361 days respectively (Table 1)

- Inflammatory cuffs increased significantly from day 81 and from day 60 up to day 361 around bronchi and vessels respectively. Cuffing infiltrates were more prominent around vessels.(Fig 1)

- The area of airway epithelium occupied by mucins was 18 and 5 times higher on days 281 and 361 post infection respectively, than measurements before day 80 in the Pneumocystisexposed rats (Fig 2)

- Cuffing inflammation and the area of epithelium occupied by mucins remained stable in the non-exposed rats (Fig 2)

- Collagen levels were not different among groups throughout the experiment.

\begin{tabular}{|c|c|}
\hline Age (Days) & $\begin{array}{c}\text { P. carinii-DNA } \\
\text { Pos./Total (\%) }\end{array}$ \\
\hline 40 & $6 / 6(100 \%)$ \\
61 & $6 / 6(100 \%)$ \\
81 & $6 / 6(100 \%)$ \\
259 & $2 / 6(33,3 \%)$ \\
361 & $1 / 6(16,7 \%)$ \\
\hline
\end{tabular}

Table 1. Detection of $P$. carinii
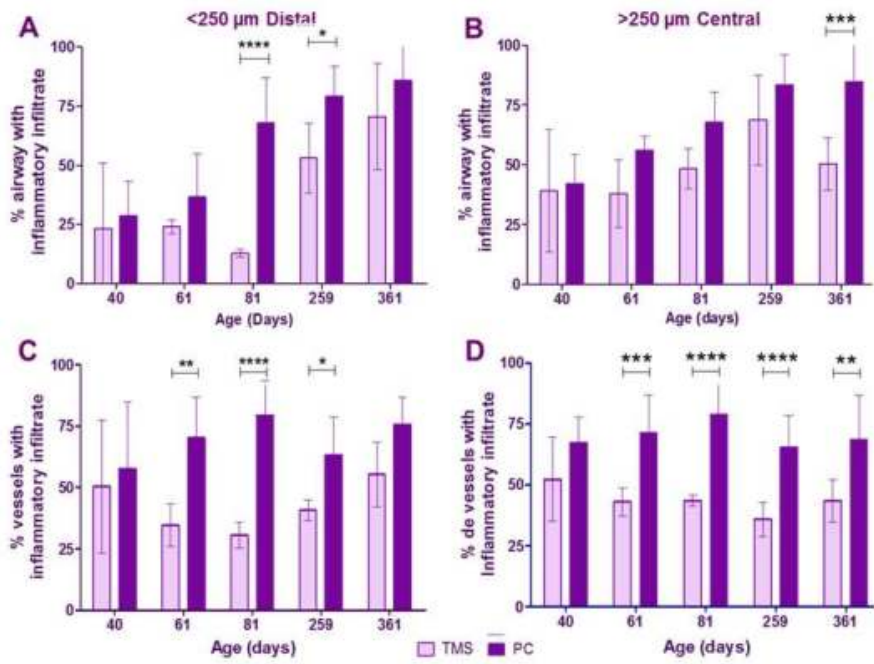

Figure 2: Percent of airways and vessels with peribronchiolar or perivascular inflammatory cuffing in rats at 40,61, 81, 259 \& 361 days of age. Using microscopy and hematoxilin-eosin stain. Airways (A \& B) and Vessels (C\& D).

\section{CONCLUSION}

- The primary infection by Pneumocystis induces long-term changes consisting of persisting peribronchial and perivascular inflammation and severely increased mucin secretion that is consistent with long-term epithelium changes of mucous cells.

- These changes support a role of Pneumocystis in chronic airway disease.

\section{References}

1. Vargas SL, Ponce CA, Gallo M, Pérez F, Astorga JF, Bustamante R, Chabé M, Durand-Joly I, Iturra P, Miller RF, Aliouat et E, Deicas E. Near-universal prevalence of Pneumocystis and associated increase in mucus in the lungs of infants with sudden unexpected death. Clin Infect Dis, 2013 Jan; 56(2): 171-9

2. Iturra PA, Rojas DA, Pérez FJ, Méndez A, Ponce CA, Bonilla P, Bustamante R, Rodríguez H, Beltrán CJ, Vargas SL. Progression of Type 2 Helper T CellType Inflammation and Airway Remodeling in a Rodent Model of Naturally Acquired Subclinical Primary Pneumocystis Infection.. Am J Pathol 2018 Feb;188(2): 417-431 